\title{
Nonlinear Evolution of a Steep, Focusing Wave Group in Deep Water Simulated with OceanWave3D
}

Barratt, Dylan ; Bingham, Harry B.; Adcock, Thomas A. A.

Published in:

Journal of Offshore Mechanics and Arctic Engineering

Link to article, DOI:

$10.1115 / 1.4044989$

Publication date:

2020

Document Version

Peer reviewed version

Link back to DTU Orbit

Citation (APA):

Barratt, D., Bingham, H. B., \& Adcock, T. A. A. (2020). Nonlinear Evolution of a Steep, Focusing Wave Group in Deep Water Simulated with OceanWave3D. Journal of Offshore Mechanics and Arctic Engineering, 142(2), [ 021201]. https://doi.org/10.1115/1.4044989

\section{General rights}

Copyright and moral rights for the publications made accessible in the public portal are retained by the authors and/or other copyright owners and it is a condition of accessing publications that users recognise and abide by the legal requirements associated with these rights.

- Users may download and print one copy of any publication from the public portal for the purpose of private study or research.

- You may not further distribute the material or use it for any profit-making activity or commercial gain

- You may freely distribute the URL identifying the publication in the public portal 


\section{NONLINEAR EVOLUTION OF A STEEP, FOCUSING WAVE GROUP IN DEEP WATER SIMULATED WITH OCEANWAVE3D}

\author{
Dylan Barratt* \\ Department of Engineering Science \\ University of Oxford \\ Oxford, Oxfordshire, OX1 3PJ, UK \\ Email: dylan.barratt@eng.ox.ac.uk
}

\author{
Harry B. Bingham \\ Department of Mechanical Engineering \\ Technical University of Denmark \\ 2800 Lyngby, Denmark \\ Email: hbb@mek.dtu.dk
}

\author{
Thomas A. A. Adcock \\ Department of Engineering Science \\ University of Oxford \\ Oxford, Oxfordshire, OX1 3PJ, UK \\ Email: thomas.adcock@eng.ox.ac.uk
}

\begin{abstract}
Steep, focusing waves can experience fast and local nonlinear evolution of the spectrum due to wave-wave interactions resulting in energy transfer to both higher and lower wavenumber components. The shape and kinematics of a steep wave may, thus, differ substantially from the predictions of linear theory. We have investigated the role of nonlinear interactions on group shape for a steep, narrow-banded, directionally-spread wave group focusing in deep water using the fully-nonlinear potential flow solver, OceanWave3D. Exact second-order correction of the initial conditions has been implemented together with a novel third-order approximate correction based on a Stokes-type formulation for surface elevation combined with a scaling argument for the third-order velocity potential. Four-phase separation reveals that the third-order scheme provides a good estimate for the third-order superharmonics. A quantitative assessment of numerical error has also been performed for the spatial and temporal discretization, including energy conservation, a reversibility check and validation against previous simulations performed with a higher-order spectral (HOS) code. The initially narrowbanded amplitude spectrum exhibits the formation of sidelobes at angles of approximately $\pm 35 \mathrm{deg}$ to the spectral peak during the simulated extreme wave event, occurring in approximately 10 wave periods, with a preferential energy transfer to highwavenumber components. The directional energy transfer is attributed to resonant third-order interactions with a discussion of the engineering implications.
\end{abstract}

\footnotetext{
*Corresponding Author
}

\section{INTRODUCTION}

The shape and kinematics of extreme waves remains a topic of interest for research and a relevant consideration for offshore engineering design. Extreme wave formation can arise from dispersive focusing, characterised by the superposition of Fourier modes, as well as various nonlinear focusing mechanisms such as the modulational instability; wave interactions with current, wind and bathymetry; crossing seas and shallow water effects with extensive reviews by Kharif \& Pelinovsky [1], Dysthe et al. [2] and Adcock \& Taylor [3]. Extreme waves are characterised by excessive steepness $\left(\varepsilon=\pi H_{0} / \lambda_{0}\right)$, defined in terms of the characteristic height $\left(H_{0}\right)$ and characteristic length $\left(\lambda_{0}\right)$ of the waves. Linear theory approximately describes the evolution of a wave group if $\varepsilon<<1$ but nonlinearity becomes significant for steeper waves due to the presence of higher-order nonlinear terms in the dynamic and kinematic free surface boundary conditions. Rogue waves are typically expected to be steep and consequently exhibit a greater prevalence of nonlinearity. The shape of an extreme wave has been previously investigated for "weakly nonlinear" random seas featuring waves of moderate steepness with a uniform distribution of phase [4-6]. Random-sea investigations of extreme waves represent the most realistic approach and account for the natural variability of focused events as well as the interaction of focused events with surrounding wave groups and the background sea state. However, the random-sea approach is also innately computationally inefficient because, for much of the space and time, the simulation is resolving wave events which are not particularly extreme. Thus, an alternate approach 


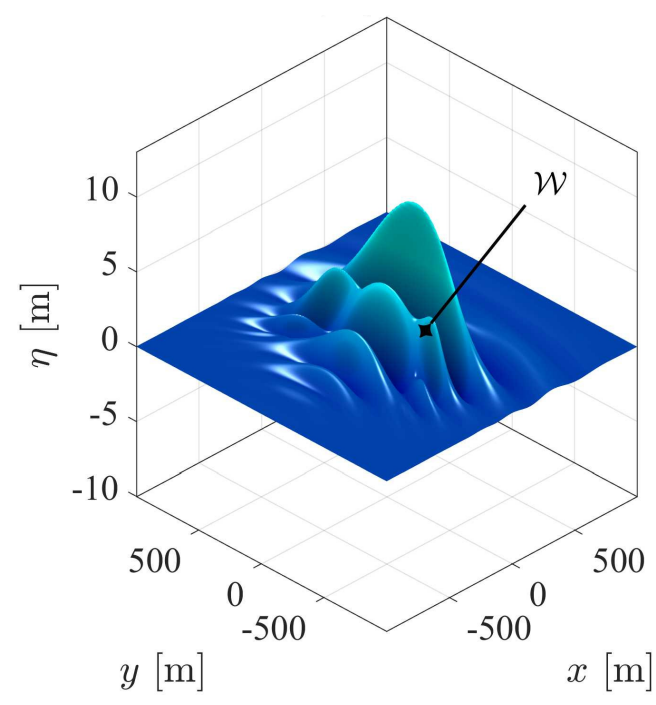

FIGURE 1. WING WAVES: constructive interference between wing waves and central crests as observed by Gibbs \& Taylor [8].

has been used, based on isolated wave groups featuring a coherent phase distribution designed to focus components in time and space; this approach has also been suggested as a design method for the assessment of loads on offshore structures [7] since a focusing wave group encompasses all forms of wave-wave interaction present in a random sea but remains less computationally expensive to simulate, less vulnerable to error wave contamination (since the error waves separate out from the wave group during the simulation) and changes in group shape can be more easily identified. Gibbs \& Taylor [8] simulated extreme waves of the NewWave form [9], with directional spreading, using the "BST" code of Bateman, Swan \& Taylor [10], with a $5^{\text {th }}$-order Dirichlet-Neumann operator, and identified third-order nonlinear wave-wave interactions as the cause of group contraction in the direction of propagation and group expansion in the lateral direction as well as the movement of the largest crest to the front of the group. The lateral group expansion has been linked to the formation of "wing waves" (labeled $\mathcal{W}$ in Fig. 1) which manifest as localised protrusions at the periphery of the group and interfere constructively with the central crests to form notably broader crests. An intriguing feature of the wing waves is the observed direction of travel, at approximately $\pm 35 \mathrm{deg}$ to the propagating wave group, leading Adcock \& Taylor [11] to suggest that the wing waves are a direct result of third-order interactions along the $\arctan (1 / \sqrt{ } 2)$ resonance angle identified by LonguetHiggins [12] for the spectral peak of a three-dimensional wave packet. The present study investigates the spectral evolution of a steep, focusing wave group, of the same form as Gibbs \& Taylor [8], using the fully-nonlinear potential flow solver OceanWave $3 D[13]$ and performs a detailed assessment of the simulation fidelity.

\section{NUMERICAL METHOD}

OceanWave $3 D$ numerically solves the governing equations of potential flow for surface gravity waves [14] in a threedimensional Eulerian frame of reference using a Cartesian coordinate system $(x, y, z)$ with the origin of the vertical coordinate $(z=0)$ at the mean-water-level. The code is, thus, restricted to free-surface shapes which can be expressed as a single-valued function of the horizontal coordinates and cannot capture overturning waves.

\section{Code Description}

The governing equations require nonlinear boundary conditions to be applied at the free surface and, as a "fully nonlinear" code, OceanWave $3 D$ applies the prescribed boundary conditions without simplification. However, the location of the free surface, $\eta(x, y, t)$, is unknown a priori. Thus, a non-conformal transform maps the solution to a time-invariant domain:

$$
\sigma \equiv \frac{z+d(x, y)}{\eta(x, y, t)+d(x, y)},
$$

compatible with a variable depth domain, $d(x, y)$, but restricted to constant depth, $d(x, y)=d$, for the present study. Discretization of the governing equations is performed with a method of lines approach, based on time integration with the classical explicit four-stage, fourth-order Runge-Kutta scheme and finitedifference discretization of the spatial derivatives with direct product approximation of the nonlinear terms. A grid $\left(N_{x}, N_{y}\right)$ of uniformly distributed points is defined along the horizontal $x y$-axes for evolution of the free-surface variables $\eta(x, y, t)$ and $\tilde{\phi}=\phi(x, y, \eta, t)$. In the $\sigma$-transformed domain, $N_{\sigma}$ points are defined in the vertical direction below each horizontal free-surface grid point, in the restricted range $0 \leq \sigma \leq 1$. Thus, $h$-adaptivity is implemented by adjusting the number of grid points in the horizontal and vertical directions, for a given size of domain, to refine or coarsen the spatial resolution. In the interior of the domain, all derivatives are centrally discretized with a flexible-order finitedifference scheme that allows for $p$-adaptivity. Near the structural boundaries (i.e., the bottom and sidewalls of the domain) stencils become off-centered but the order of the scheme is the same as the interior domain. Simultaneous application of the Laplace equation and no-normal-flow condition is accomplished at the structural boundaries using a single layer of ghost nodes outside the physical domain of interest, as explained by EngsigKarup et al. [13]. Discretization, thus, yields a rank $n=N_{x} N_{y} N_{z}$ linear system of equations of the form:

$$
A \Phi=b,
$$

comprised of the coefficient matrix $A$, the unknown vector of velocity potential $(\Phi)$ corresponding to each grid point at an in- 
stant in time and the vector $b$ holding zeros and the inhomogeneous boundary conditions. Numerical solution of Eq. (2) is performed with the Generalized Minimum Residual (GMRES) method with left-preconditioning by the linearised version of the coefficient matrix $A$, constructed with a second-order finite difference scheme and denoted as $A_{2}$ :

$$
A_{2}^{-1}\{A \Phi=b\}
$$

The preconditioner $A_{2}$ is not time dependent and, thus, needs only to be generated once at the beginning of the simulation. Based on an initial guess for $\Phi_{0}$, the initial residual is computed, $r_{0}=A \Phi_{0}-b$, and preconditioned by solving $A_{2} u_{0}=r_{0}$ followed by one iteration of the GMRES algorithm in the Krylov subspace. Preconditioning is, thus, repeated once every iteration solving a system of the form $A_{2} u_{m}=r_{m}$ where $m$ indicates the iteration number. Optimal linear scaling of the solution effort is ensured by multi-grid solution of the preconditioning step using Gauss-Seidel smoothing. The preconditioner $A_{2}$ is thus generated for every grid level using Direct Coarse grid Approximation (DCA) and a V-cycle with one pre-smoothing step and one post-smoothing step employed. The number of GMRES iterations required for convergence at a particular time step has been found to be independent of grid size using the multi-grid preconditioning scheme [13]. Notably, the inclusion of ghost nodes has also been shown to improve the numerical stability of the preconditioning relaxation scheme, especially in the presence of the Neumann boundary conditions applied at the structural boundaries [13], allowing for an anisotropic grid distribution in the vertical direction. Clustering of points near the free surface using Chebyshev-Gauss-Lobatto (CGL) points has, thus, been implemented to minimise dispersion error, anticipated to be the dominant form of error in OceanWave3D [15].

\section{Numerical Domain}

A numerical wave tank $7680 \mathrm{~m}$ in length $(L), 2560 \mathrm{~m}$ in width $(W)$ and $200 \mathrm{~m}$ in depth $(d)$ has been employed. The simulated extreme wave event follows the dimensions and timescales of Gibbs \& Taylor [8] with a characteristic wavelength $\left(\lambda_{p}\right)$ of $225 \mathrm{~m}$ and characteristic period $\left(T_{p}\right)$ of $12 \mathrm{~s}-$ both corresponding to the initial peak of the wavenumber spectrum, $k_{p}=0.02796 \mathrm{~m}^{-1}$. The water is consequently approximated as "deep" with $k_{p} d=5.6$. A symmetry plane has been implemented in the transverse $(y)$ direction along the center plane of the group to exploit the symmetry property of the wave event (see Fig. 1).

TABLE 1. Initial Condition Parameters.

\begin{tabular}{|c|c|c|c|}
\hline$A k_{p}$ & $k_{p}$ & $k_{w}$ & $\varsigma$ \\
\hline \hline 0.3 & $0.02796 \mathrm{~m}^{-1}$ & $0.004606 \mathrm{~m}^{-1}$ & $15^{\circ}$ \\
\hline
\end{tabular}

\section{INITIAL CONDITIONS}

The NewWave model describes the average shape of an extreme wave in a linear sea using the scaled autocorrelation function, as detailed by Tromans et al. [9]. In the present study, initial conditions for the simulations have been prescribed as an approximation for a NewWave group calculated at 20 wave periods before the linear focus time, using the linear dispersion relationship. The low steepness of the dispersed initial conditions minimises the contribution of higher-order bound harmonics. However, exact second-order correction of the surface elevation and velocity potential has been performed with the expressions of Dalzell [16] for finite-depth wave-wave interactions - the second order terms have been evaluated at the mean-water-level $(z=0)$ but the first-order terms have been evaluated at the free surface $(z=\eta)$ to avoid pseudo-second-order error waves, as detailed in Appendix A. Time marching of the initial conditions has been performed for a total of 40 wave periods, terminating the simulation at 20 wave periods after the linear focus time. The parameters for the initial conditions of the wave group match those of Gibbs \& Taylor [8] and are listed in Table 1.

Conventionally, directional spreading of the frequency spectrum is implemented as the product of a unidirectional spectrum, $S(\omega)$, and a spreading function, $D(\theta)$, where $\omega$ is the angular frequency and $\theta$ the direction of wave propagation: $F(\omega, \theta)=S(\omega) D(\theta)$.

A Gaussian unidirectional spectrum has been implemented in terms of wavenumber, $\hat{S}(k)$, by this study:

$$
\hat{S}(k)=\exp \left(\frac{-\left(k-k_{p}\right)^{2}}{2 k_{w}^{2}}\right)
$$

where $k$ is the wavenumber, $k_{p}$ is the wavenumber corresponding to the initial spectral peak and $k_{w}$ is the spectral width, which yields a close approximation to a JONSWAP spectrum with a peak enhancement of $\gamma=3.3$. A Gaussian distribution has also been used for the spreading function:

$$
D(\theta)=\frac{1}{\sqrt{2 \pi} \varsigma} \exp \left(-\left(\frac{\theta^{2}}{2 \varsigma^{2}}\right)\right)
$$

with a constant rms spreading parameter $(\varsigma)$ of $15 \mathrm{deg}$. The directional frequency spectrum, $F(\omega, \theta)$, has thus been defined as the product of two Gaussian functions so the surface elevation of the focused linear event assumes an approximate form expressed in terms of the spatial bandwidths $S_{x}$ and $S_{y}$ :

$$
\eta(x, y)=A e^{-\frac{1}{2} S_{x}^{2} x^{2}} e^{-\frac{1}{2} S_{y}^{2} y^{2}} \cos \left(k_{p} x\right),
$$

which represents a close approximation to the form of a linearlyfocused NewWave group. 


\section{Phase Separation Technique}

A system of bound harmonics associated with each free harmonic can be described with a Stokes regular wave expansion, shown here to the fourth-order for a single amplitude component, $a_{i}$, with phase $\vartheta_{i}(t)$ :

$$
\begin{aligned}
\eta_{i}(t)= & +a_{i} \cos \vartheta_{i}(t) \\
& +C_{22} a_{i}^{2} \cos 2 \vartheta_{i}(t)+C_{20} a_{i}^{2} \\
& +C_{31} a_{i}^{3} \cos \vartheta_{i}(t)+C_{33} a_{i}^{3} \cos 3 \vartheta_{i}(t) \\
& +C_{42} a_{i}^{4} \cos 2 \vartheta_{i}(t)+C_{44} a_{i}^{4} \cos 4 \vartheta_{i}(t),
\end{aligned}
$$

where $\vartheta_{i}(t)=k_{i} x \cos \theta_{i}+k_{i} y \sin \theta_{i}-\omega_{i} t+\varphi_{0}$. The initial phase $\varphi_{0}$ is defined relative to a 0 deg baseline and the coefficients, $C$, determine depth sensitivity, as listed by Walker et al. [17]. The method of phase separation, e.g. Fitzgerald et al. [18], allows particular terms of the Stokes expansion to be isolated by performing simulations with an offset in the initial phase, $\varphi_{0}$, followed by addition/subtraction of the results. Performing simulations with $0 \mathrm{deg}$, $90 \mathrm{deg}, 180 \mathrm{deg}$ and $270 \mathrm{deg}$ phase shifts for the initial phase allows for isolation of the first-order free harmonic and the third-order principal harmonic (generally considered negligible) to obtain the linearised surface elevation $\left(\eta_{L}\right)$ :

$$
\eta_{L}(t)=a_{i} \cos \vartheta_{i}(t)+C_{31} a_{i}^{3} \cos \vartheta_{i}(t)
$$

Linearisation of the wave spectrum has, thus, been performed with the four-phase separation technique in this study.

\section{Approximate Third-Order Correction}

An exact description of the third-order bound harmonics has been presented by Madsen \& Fuhrman [19]. However, an approximate description may be sufficient for the present study since the third-order harmonics are small for the initially dispersed group. An approximation has been proposed by Walker et al. [17] based on the Stokes-type expansion in Eq. (7) with use of the Hilbert transform, denoted by $\mathcal{H}$, which introduces a 90deg phase shift into the operand. The third-order principal harmonic of surface elevation $\left(\eta_{31}\right)$ can, thus, be approximated as:

$$
\eta_{31}=\frac{-3\left(1+3 B+3 B^{2}+2 B^{3}\right)}{8(1-B)^{3}} k_{0}^{2} \eta_{L}\left(\eta_{L}^{2}+\left[\mathcal{H}\left\{\eta_{L}\right\}\right]^{2}\right),
$$

and the third-order superharmonic of surface elevation $\left(\eta_{33}\right)$ as:

$$
\eta_{33}=\frac{3\left(1+3 B+3 B^{2}+2 B^{3}\right)}{8(1-B)^{3}} k_{0}^{2} \eta_{L}\left(\eta_{L}^{2}-3\left[\mathcal{H}\left\{\eta_{L}\right\}\right]^{2}\right),
$$

where $B=\operatorname{sech}\left(2 k_{0} d\right)$ and $k_{0}$ is the characteristic wavenumber.

The corresponding velocity potential associated with the third-order surface elevation has been approximated in this study with a scaling argument. Noting the scaling relation between velocity potential and surface elevation $\phi \sim g \eta / \omega$ (described by Lannes [20]) and the 90deg phase shift expected between surface elevation and velocity potential, an approximation can, thus, be obtained for the third-order superharmonic:

$$
\phi_{33}=K_{33} \frac{g}{\omega_{0}} \mathcal{H}\left\{\eta_{33}\right\}
$$

where $\omega_{0}$ is the characteristic frequency and the coefficient $K_{33}$ is a proportionality constant. A similar expression can be obtained for the third-order principal harmonic and both sets of third-order terms have been assessed in this study as a proposed method for correction of the initial conditions, with the proportionality constants set to unity. Simplified expressions for the bound harmonics can also be found in Adcock \& Taylor [11] with detailed comparisons against fully-nonlinear simulation results.

\section{SIMULATION FIDELITY}

A convergence study based upon grid resolution ( $h$ adaptivity) has been performed with the use of grid-halving in all three dimensions to assess grid independence across two resolution levels, termed "intermediate" and "fine", both listed in Table 2 with the height of the first grid at the free surface $\left(\Delta z^{*}\right)$ and the grid resolution of Gibbs \& Taylor [8], denoted as G\&T'05, also listed. A convergence study based upon $p$-adaptivity has also been performed by altering the order of the finite difference scheme used for the spatial derivatives in the governing equations. Time integration of the governing equations is performed with the classic fourth-order Runge-Kutta scheme and sensitivity to time step size $(\Delta t)$ has been assessed with the Courant-Friedrichs-Lewy (CFL) condition calculated from the phase speed associated with the initial peak of the wavenumber spectrum. All simulations have been performed with exact second-order correction of the initial conditions; approximate third-order correction has been implemented where stated. The validity of the "deep" water assumption has also been assessed together with the modelling error of the proposed third-order correction scheme for the initial conditions.

TABLE 2. Discretization Parameters.

\begin{tabular}{|c|c|c|c|c|c|c|}
\hline Grid & $N_{x}$ & $N_{y}$ & $N_{z}$ & $\Delta x$ & $\Delta y$ & $\Delta z^{*}$ \\
\hline \hline Fine & 1025 & 257 & 17 & $7.5 \mathrm{~m}$ & $10 \mathrm{~m}$ & $0.25 \mathrm{~m}$ \\
\hline Inter. & 513 & 129 & 9 & $15 \mathrm{~m}$ & $20 \mathrm{~m}$ & $1 \mathrm{~m}$ \\
\hline G\&T'05 & 255 & 127 & - & $14.1 \mathrm{~m}$ & $22.7 \mathrm{~m}$ & - \\
\hline
\end{tabular}


TABLE 3. Average Number of GMRES Iterations.

\begin{tabular}{|c|c|c|c|c|c|c|}
\hline Grid & CFL $_{\mathrm{x}}$ & $n_{x}$ & $n_{y}$ & FD4 & FD6 & FD8 \\
\hline \hline Fine & 0.5 & 30 & 22.5 & 19.34 & 21.10 & 23.19 \\
\hline Inter. & 0.5 & 15 & 11.3 & 13.69 & 15.64 & 23.15 \\
\hline
\end{tabular}

\section{Order of Finite Differencing}

OceanWave $3 D$ utilises centered stencils for the finite difference discretization of spatial derivatives in the governing equation, except near the structural boundaries where off-center stencils must be used. Thus, only even-numbered orders can be implemented and the current study has performed simulations with fourth, sixth and eighth-order finite difference schemes on both the intermediate and fine grids. The maximum number of multigrid levels $\left(N_{M G}\right)$ has been used for preconditioning on both the fine grid, $N_{M G}=10$, and the intermediate grid, $N_{M G}=9$. A CFL of 0.5 has previously been shown to be adequate for steep waves [13] and has also been used here. Increasing the order of the finite difference scheme has also been shown to improve dispersion error without significantly influencing diffusion error [15]. Since OceanWave $3 D$ is anticipated to be dispersion-error dominant, $p$-adaptivity is, thus, a particularly effective method of improving simulation fidelity. A key feature of OceanWave $3 D$ is the grid-independent iteration count for the GMRES algorithm, with multi-grid preconditioning, which ensures optimal scaling of the solution effort. The average number of GMRES iterations required for convergence at each time step, with a tolerance of $10^{-6}$ (see Table I of [21]), has been recorded over the entirety of each simulation, from $-20 T_{p}$ to $+20 T_{p}$, and listed in Table 3 for both the intermediate and fine grid resolutions. The number of grid points per characteristic wavelength in the direction of wave group propagation, $n_{x}=\lambda_{p} / \Delta x$, and the direction transverse to propagation, $n_{y}=\lambda_{p} / \Delta y$, has also been listed for both grid resolutions. As can be seen, a grid-independent iteration count arises for eighth-order finite differencing but not for the sixth-order and fourth-order schemes. Simulations of steep waves with OceanWave $3 D$ have been previously shown to require a grid resolution of at least $n_{x} \approx 32$ with sixth-order finite differencing [13]. The low number of GMRES iterations on the intermediate grid with sixth and fourth-order schemes may, thus, be a result of inadequate spatial resolution for the given discretization schemes. Eighth-order finite difference schemes have, thus, been used for the present study on both the intermediate and fine grids.

\section{Grid Convergence and CFL}

The peak surface elevation $\left(\eta_{\text {peak }}\right)$ occurring at any point in the simulation has been recorded for both grid levels, intermediate and fine, for CFL conditions of 0.5 and 1.0. Similarly, the peak vertical velocity $\left(\tilde{w}_{\text {peak }}\right)$ and the peak horizontal velocity $\left(\tilde{u}_{\text {peak }}\right)$ have also been recorded to assess convergence of the kinematics. Refinement of the grid resolution has been shown to suppress both diffusion and dispersion errors [15]; thus, $h$-adaptivity offers an effective convergence strategy for OceanWave3D. Refinement of the time step to reduce the CFL condition, for a given grid resolution, has been shown to suppress diffusion error but not dispersion error. Since, by definition, kinematics are expressed in terms of derivatives of the velocity potential, the order of convergence for the kinematics is expected to be lower than that of the velocity potential and surface elevation. The results of the convergence study are listed in Table. 4 and compared with the results of Gibbs \& Taylor [8] for the same test case. The fine grid simulation performed with a CFL of 0.5 using OceanWave $3 D$ is expected to be converged since the CFL condition and the horizontal resolution of $n_{x}=30$ meets the recommendation for steep, nonlinear waves [13] - this case has, thus, been compared with the results of Gibbs \& Taylor [8] using the time history of surface elevation at the location of nonlinear focus plotted in Fig. 2. Nonlinear focus occurs at $t=1.3 T_{p}$, relative to the linear focus time of $t=0 T_{p}$, indicating a slight delay in the focusing event due to nonlinear wave-wave interactions. Cubic spline interpolation has been used on both curves to improve the plotting resolution. Good agreement between the time histories can be seen, although a slight lead by OceanWave $3 D$ is observed before focus and a slight lead by the HOS code is observed after focus. Table 4 indicates a $0.3 \%$ discrepancy in $\eta_{\text {peak }}$ between Gibbs \& Taylor [8] and the fine-grid result with a CFL of 0.5 which further supports the conclusion of convergence. In contrast, diffusion error seems apparent on the intermediate grid with a CFL condition of 1.0 since all the convergence parameters are substantially lower than the converged fine grid result with a CFL of 0.5 . The intermediate grid with a CFL of 0.5 , however, indicates an deviation of $-2.6 \%$ for $\eta_{\text {peak }},-2.3 \%$ for $\tilde{w}_{\text {peak }}$ and $-10.1 \%$ for $\tilde{u}_{\text {peak }}$ which may be seen as an unexpectedly good result since

TABLE 4. Convergence study based on grid resolution ( $h$-adaptivity) and CFL with eighth-order finite differencing (FD8).

\begin{tabular}{|c|c|c|c|c|c|c|}
\hline Grid & $\mathrm{CFL}_{\mathrm{x}}$ & $n_{x}$ & $n_{y}$ & $\eta_{\text {peak }}$ & $\tilde{w}_{\text {peak }}$ & $\tilde{u}_{\text {peak }}$ \\
\hline & {$[-]$} & {$[-]$} & {$[-]$} & {$[\mathrm{m}]$} & {$[\mathrm{m} / \mathrm{s}]$} & {$[\mathrm{m} / \mathrm{s}]$} \\
\hline \hline Inter. & 1.0 & 15 & 11.3 & 11.725 & 5.435 & 7.405 \\
\hline Inter. & 0.5 & 15 & 11.3 & 12.466 & 5.689 & 8.075 \\
\hline Fine & 1.0 & 30 & 22.5 & 12.702 & 5.767 & 8.837 \\
\hline Fine & 0.5 & 30 & 22.5 & 12.801 & 5.820 & 8.980 \\
\hline Fine, $N_{z}=9$ & 0.5 & 30 & 22.5 & 12.786 & 5.810 & 8.954 \\
\hline G\&T'05 & 0.13 & 16 & 9.9 & 12.840 & - & - \\
\hline
\end{tabular}




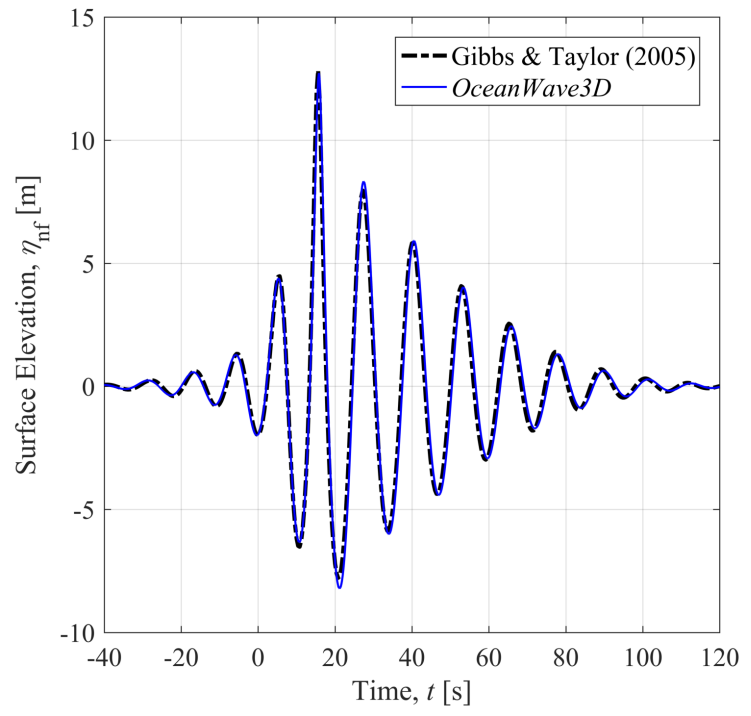

FIGURE 2. VALIDATION: time history of surface elevation at nonlinear focus point-comparison between OceanWave3D and HigherOrder Spectral (HOS) results of Gibbs \& Taylor [8].

the total grid size has been reduced by a factor of 8 . The fine grid resolution with a CFL of 1.0 exhibits deviations of $-0.8 \%$ for $\eta_{\text {peak }},-0.9 \%$ for $\tilde{w}_{\text {peak }}$ and $-1.6 \%$ for $\tilde{u}_{\text {peak }}$ which suggests that the physical parameters are reasonably well resolved on the fine grid with a CFL of 1.0. However, the diffusivity of the solution can be assessed with the total energy of the solution $(E)$ at every time step, including kinetic energy and potential energy contributions. Potential flow is inherently non-diffusive since the combination of the incompressibility condition, $\nabla \cdot u=0$, and the irrotationality condition, $\nabla \times u=0$, identically satisfies $\nabla^{2} u=0$ while the assumption of an inviscid fluid theoretically eliminates viscous dissipation. However, discretization of the governing equations with finite differencing results in numerical diffusion, due to truncation error, which can also mimic the effects of dissipation and reduce the total energy of the wave field. The change in total energy relative to the initial condition is shown in normalised form in Fig. 3 for the cases in Table. 4. As can be seen, the fine grid resolution with a CFL of 0.5 exhibits a change in total energy of $-0.024 \%$ over the complete simulation. The change in total energy occurs predominantly during the nonlinear focus event, from $0 T_{p}$ to $10 T_{p}$, when the wave group is steepest and numerical diffusion likely to be most severe. A decline in total energy is also expected as a result of the Savitzky-Golay filters which prevent the accumulation of high-frequency aliasing errors in OceanWave $3 D$. In contrast, the fine grid solution with a CFL of 1.0 exhibits a total energy change of $-0.63 \%$ which is more than an order of magnitude greater and even exceeds the $-0.46 \%$ change in total energy for the intermediate grid with a CFL of 0.5. Combining the intermediate grid with a CFL condition of
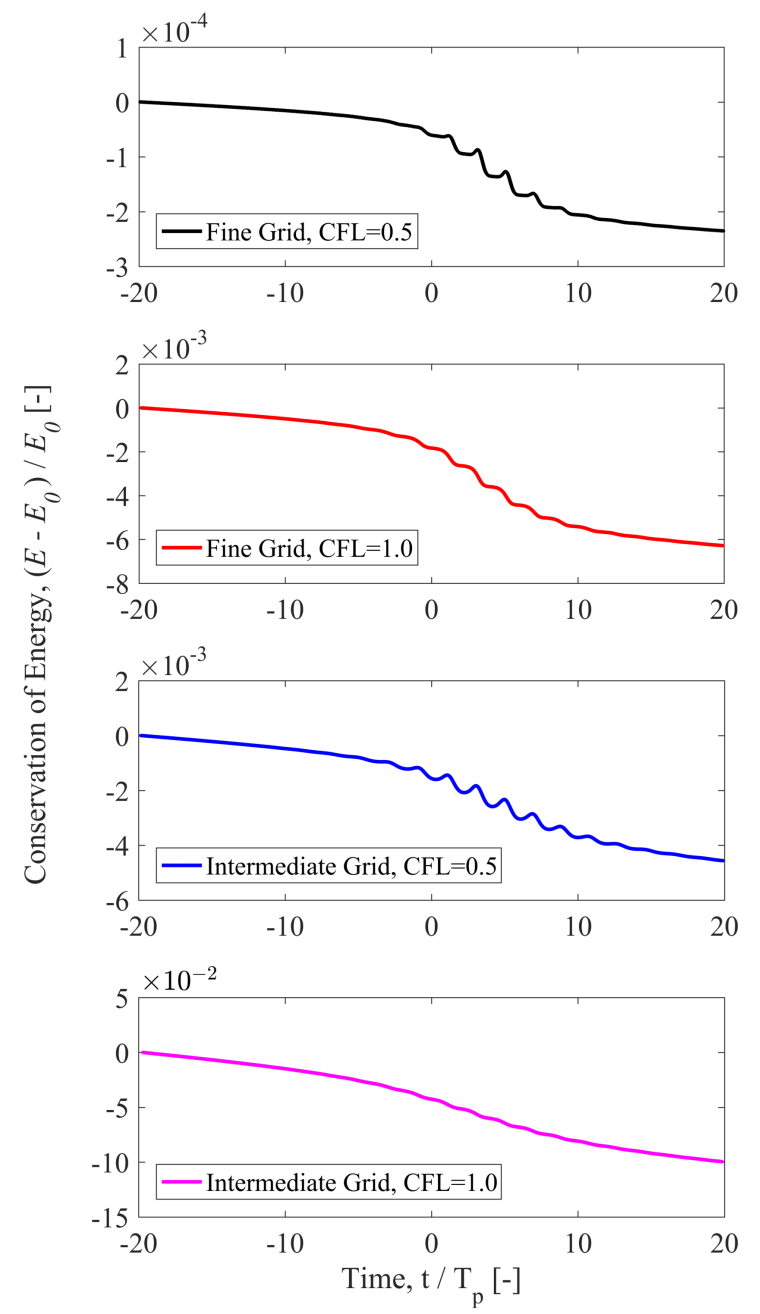

FIGURE 3. ENERGY CONSERVATION: change in total energy during simulation for various grid resolutions and CFL conditions.

1.0 further exacerbates diffusivity with a change in total energy of $-9.96 \%$ over the course of the simulation. The sensitivity of diffusion error to the CFL condition is, thus, apparent and a CFL of 0.5 has been selected for all further simulations and the results which form the basis of the discussion. A further assessment of the necessary grid resolution in the vertical $(z)$ direction has also been performed by retaining the "fine" grid resolution in the horizontal directions $\left(N_{x}=1025, N_{y}=257\right)$ while reducing the number of grid points in the vertical direction to $N_{z}=9$, with the results listed in Table. 4. As can be seen, halving the number of vertical grid points has negligibly influenced the results of the fine grid resolution with discrepancies of $-0.12 \%,-0.17 \%$, and $-0.29 \%$ in $\eta_{\text {peak }}, \tilde{w}_{\text {peak }}$ and $\tilde{u}_{\text {peak }}$, respectively, compared against the fine grid resolution with $N_{z}=17$. Thus, the reduced vertical resolution offers an attractive alternative and the implications for dispersion error are considered in the next section. 


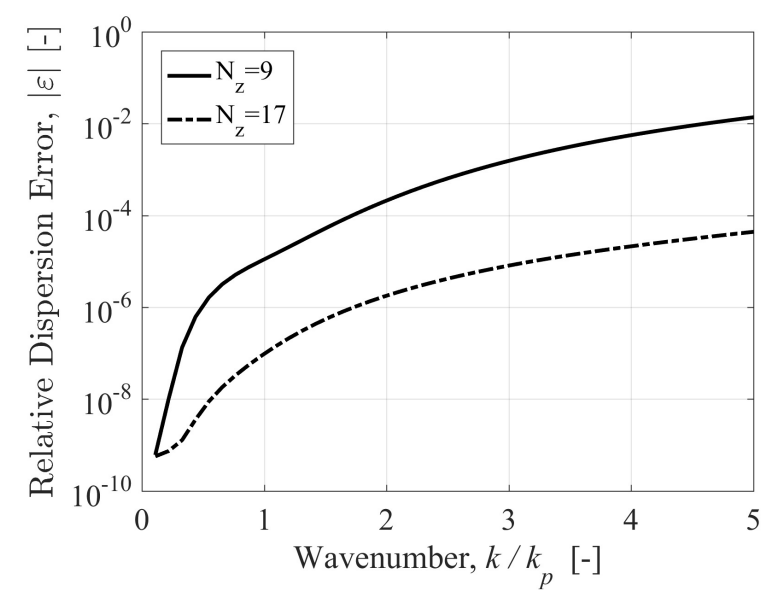

FIGURE 4. DISPERSION ERROR: linear analysis of dispersion error due to discretization of governing equations.

\section{Linear Dispersion Error}

Grid points have been clustered near the free surface using the symmetric half of a Chebyshev-Gauss-Lobatto distribution: $\sigma_{j}=\sin \left((\pi[j-1]) /\left(2\left[N_{z}-1\right]\right)\right)$, which offers a compromise between the accuracy of dispersion and the accuracy of internal kinematics [13]. Here, $j$ denotes the index of the grid point with $j=1$ at the bottom and $j=N_{z}$ at the free surface. A linear analysis of dispersion error has been performed for the fine grid resolution in the horizontal directions $\left(N_{x}=1025, N_{y}=257\right)$ while varying the the number of grid points in the vertical direction, following the method of [13] and [15], for $N_{z}=9$ and $N_{z}=17$ with a depth $(d)$ of $200 \mathrm{~m}$. The dispersion error is estimated with the unidirectional travelling wave solution, formulated in a periodic domain in $x$ with uniform transverse grid spacing $\Delta x$. The known non-dimensional dispersion operator for this solution:

$$
\frac{\tilde{w}}{k \tilde{\phi}}=\tanh k h,
$$

can be evaluated numerically on the left-hand side by applying the prescribed velocity potential at the free surface $(\tilde{\phi})$ followed by numerical solution for the vertical velocity at the free surface $(\tilde{w})$ using the eighth-order numerical scheme of OceanWave $3 D$ with linearised free surface boundary conditions. The difference between the numerically-evaluated left-hand side of Eq. (12) and the exact right-hand side of Eq. (12) yields the relative dispersion error $(\varepsilon)$, with the results shown in Fig. 4. As can be seen, both vertical resolutions exhibit negligible dispersion error for the spectral peak $\left(k / k_{p}=1\right)$. Comparatively larger errors are observed for higher wavenumbers with an error of $1 \%$ for $N_{z}=9$ at $k / k_{p}=5$. However, for the narrow-banded simulations in this study, negligible energy is associated with wavenumbers higher than $k / k_{p}=3$ suggesting than the vertical resolutions of $N_{z}=9$ and $N_{z}=17$ result in maximum dispersion errors of $0.15 \%$ and $0.001 \%$, respectively. A vertical resolution of $N_{z}=17$ has been used for the fine grid in this study, but the resolution of $N_{z}=9$ is also likely to be adequate for many engineering applications.

\section{Symmetry Plane}

A symmetry plane has been utilised along the center plane of the focusing wave group to reduce the size of the numerical domain. The boundary conditions of the symmetry plane are the same as all other sidewalls: no flow normal to the boundary enforced with a Neumann-type boundary condition in terms of the velocity potential. OceanWave $3 D$ incorporates a single layer of ghost nodes, outside the domain of interest, along all sidewalls and the bottom of the domain. Consequently, the stencils used at the sidewalls and bottom must be asymmetric for any finitedifference scheme higher than second-order. The current study utilises fourth, sixth and eighth-order finite difference schemes. Thus, asymmetric stencils are required along all boundaries (with the exception of the free surface) including the symmetry plane. Since asymmetric stencils are known to aggravate numerical diffusion, we have analysed the influence of the symmetry plane using the resolution of the intermediate grid with an eighth-order finite-difference scheme-stencil asymmetry is most severe for the case of an eighth-order finite difference scheme and the lower spatial resolution of the intermediate grid is anticipated to further exacerbate numerical diffusion. Simulations have been completed with and without a symmetry plane followed by a comparison of the surface elevation across the entire numerical domain at every time step. A maximum discrepancy of $0.13\left(10^{-3}\right) \mathrm{m}$ has been observed at a node on the symmetry plane coinciding with a crest of height $11.79 \mathrm{~m}$ - the percentage error of $0.001 \%$, thus, confirms the negligible influence of the symmetry plane.

\section{Reversibility Check}

Aliasing error due to discretization is known to accumulate amongst high frequency components. To ensure that the evolution of the amplitude spectrum (see Fig. 5) is solely the result of physical processes, a reversibility check has been performed with the enumerated steps:

1. Prescribe initial conditions at $t=-20 T_{p}$

2. Run simulation forwards in time for $32 T_{p}$

3. Halt simulation at $t=12 T_{p}$

4. Reverse sign of time step

5. Run simulation backwards in time for $32 T_{p}$

6. Halt simulation at $t=-20 T_{p}$

7. Compare forwards \& backwards simulation results

The amplitude spectrum has been calculated with a Discrete Fourier Transform (DFT) of the surface elevation, extracted from the simulation at every time step and linearised using four-phase separation. The linearised wavenumber amplitude spectrum is shown in Fig. 5 for the initial condition, $t=-20 T_{p}$, as well as the time of nonlinear focus, $t=1.3 T_{p}$, and the final condition, $t=$ 
Prescribed Initial Condition, $t=-20 T_{p}$

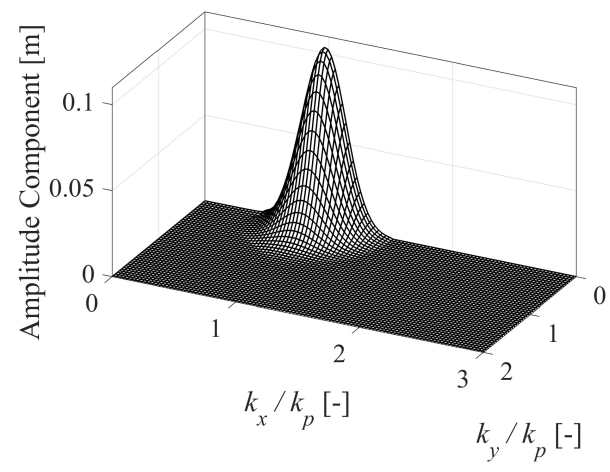

(a)

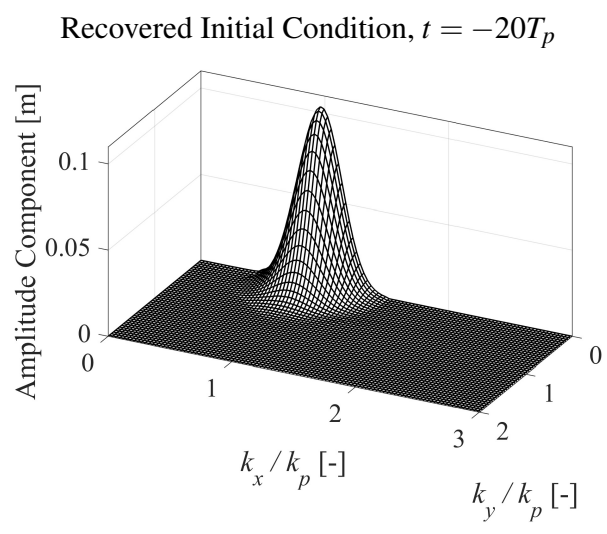

(e)

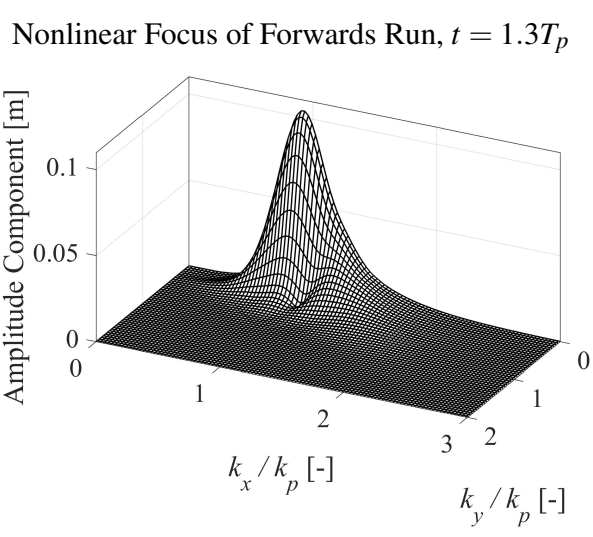

(b)

Nonlinear Focus of Backwards Run, $t=1.3 T_{p}$

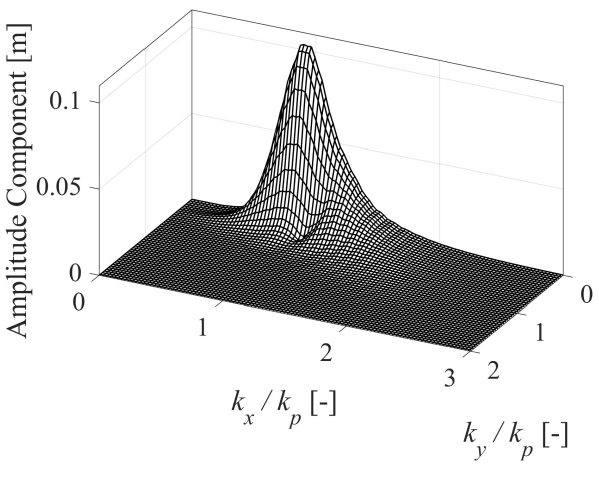

(d)

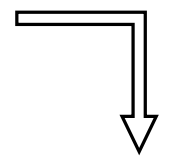

Final Condition of Forwards Run, $t=32 T_{p}$, and Initial Condition of Backwards Run

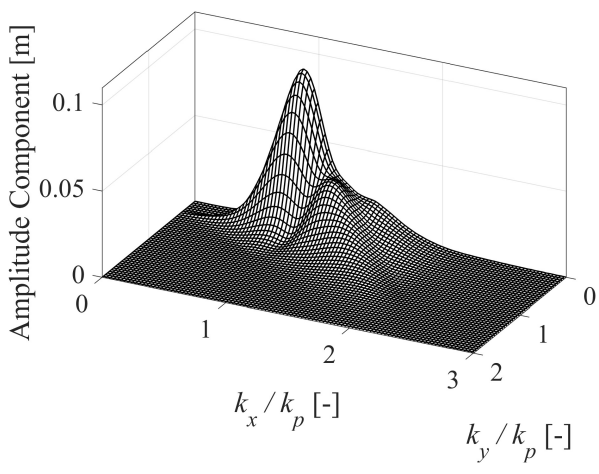

(c)

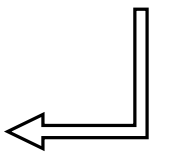

FIGURE 5. AMPLITUDE SPECTRA OF SURFACE ELEVATION: $(a-c)$ forwards run with positive time step; $(c-e)$ subsequent backwards run with negative time step to recover initial condition.

$12 T_{p}$. The initial amplitude spectrum, Fig. 5(a), depicts the prescribed Gaussian unidirectional spectrum with Gaussian directional spreading $(\varsigma)$ of $15 \mathrm{deg}$. At nonlinear focus, Fig. 5(b), the amplitude spectrum exhibits minor sidelobes-symmetrically located about the spectral peak with a discernible preferential excitation of higher wavenumbers. After nonlinear focus, Fig. 5(c), the spectral sidelobes have become markedly more pronounced, with an obvious bias towards high-wavenumber components. An appreciable downshift in wavenumber for the spectral peak can also be seen. The spectral evolution of the amplitude spectrum, thus, closely matches the observations of Gibbs \& Taylor [8] for the forwards run, indicating consistent results between the code of Bateman, Swan \& Taylor [10] and OceanWave3D. The backwards simulation depicts close agreement at nonlinear focus, Fig. $5(d)$, with the forwards simulation and eventually recovers the initial condition, Fig. 5(e), with a complete reversal of the spectral changes observed in the forwards simulation. Thus, the spectral changes are attributed entirely to physical processes of wavewave interaction rather than the accumulation of high-frequency aliasing errors.

\section{Deep Water Assumption}

The potential flow solver OceanWave $3 D$ requires a numerical domain of finite extent and, thus, cannot simulate surface waves in infinitely deep water. Dalzell [16] provides a finitedepth version of second-order theory that considers two intersecting wave trains (denoted with subscripts 1 and 2) and provides expressions for both surface elevation and velocity potential. The expressions of Dalzell [16] include second-order superharmonics and subharmonics neither of which adhere to the dispersion relation and both of which propagate with a dynamic slaved to the free wave components. The superharmonics manifest with an effective wavenumber of $k_{1}+k_{2}$ and, thus, appear as a short-wavelength modification to the free harmonics. Conversely, the subharmonics manifest with an effective wavenumber of $k_{1}-k_{2}$ and, thus, appear as a long-wavelength modification to the free harmonics. The larger length scale of the subharmonics results in greater depth sensitivity than observed for the superharmonics or free harmonics influencing the evolution of the wave group (Fig. 6). The second-order subharmonics are also associated with the formation of a return current, depicted 


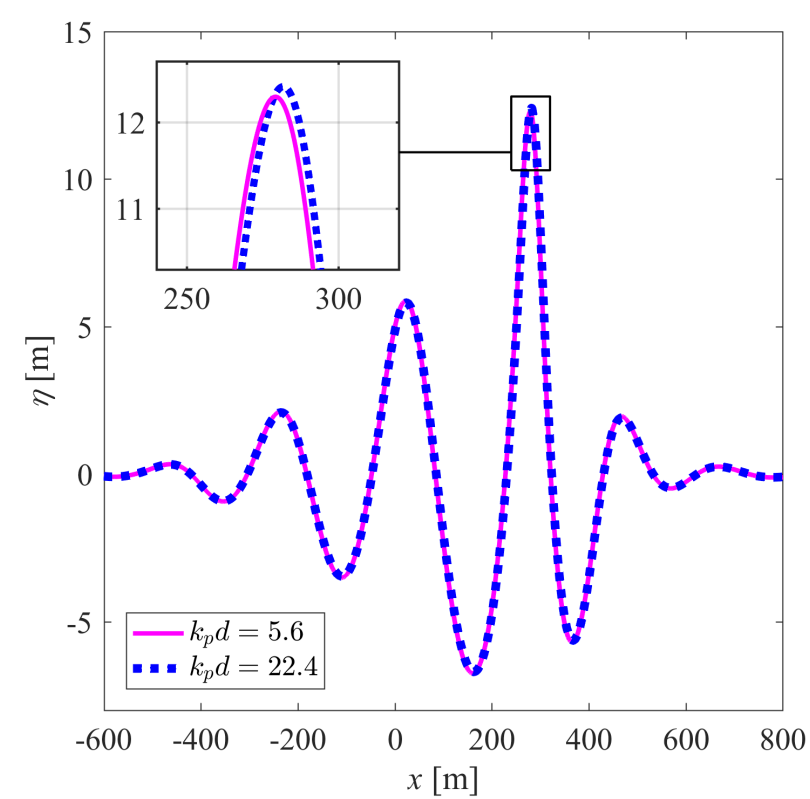

FIGURE 6. DEPTH EFFECT: surface elevation along center plane of wave group at a time $\left(t=1 T_{p}\right)$ soon before nonlinear focus for domain depths of $200 \mathrm{~m}\left(k_{p} d=5.6\right)$ and $800 \mathrm{~m}\left(k_{p} d=22.4\right)$.

in Fig. 7, beneath the wave group which counteracts the Stokes transport occurring at the free surface and forms a localised depression in surface elevation known as a set down [22]. The return current and set down scale with the dimensions of the wave group and, thus, may be influenced by finite-depth effects even in water typically approximated to be "deep" [22]. The current study has utilised a domain depth of $200 \mathrm{~m}$ which corresponds to a dimensionless depth of $k_{p} d=5.6$. To assess the significance of the finite-depth effect, a simulation has also been performed with a domain depth of $800 \mathrm{~m}$, corresponding to a dimension-
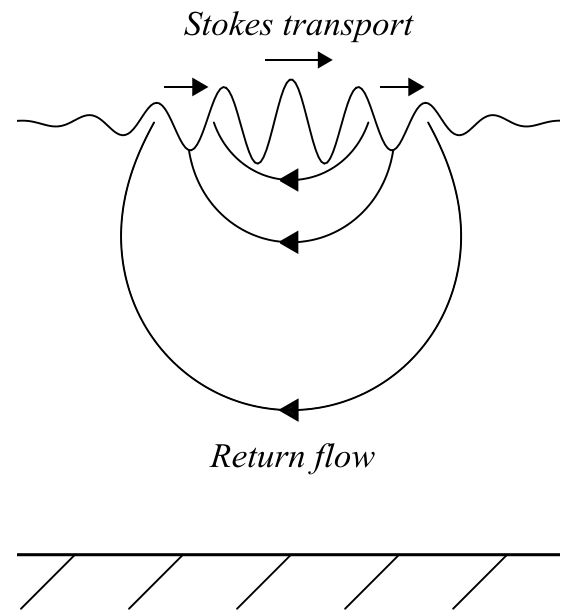

FIGURE 7. RETURN CURRENT: Stokes transport at free surface counteracted by return current underneath wave group [22]. less depth of $k_{p} d=22.4$, with a comparison of surface elevation along the center plane of the wave group at a time, $t=1 T_{p}$, soon before nonlinear focus. Cubic-spline interpolation of the curves in Fig. 6 has been implemented for clarity. The amplitude of the largest crest is $0.9 \%$ smaller for $k_{p} d=5.6$ which is expected because the set down should be more prevalent for $k_{p} d=5.6$. The largest crest in the $k_{p} d=5.6$ solution also appears to lag behind the largest crest in the $k_{p} d=22.4$ solution which is the net result of counteracting effects influencing the group velocity and return current. The group velocity is expected to be greater for $k_{p} d=5.6$, however, the velocity of the return current is also expected to be greater for $k_{p} d=5.6$ and serves to retard the propagation of the group since the direction of the return current opposes the direction of wave group propagation. Figure 6 confirms that the effect of the return current prevails causing the wave group to lag behind in the $k_{p} d=5.6$ solution. Based on Fig. 6, the depth effect is, however, considered to be negligible and $k_{p} d=5.6$ a reasonable approximation for deep water.

\section{Third-Order Error Waves}

An approximation for the third-order bound harmonics has been proposed for correction of the initial conditions, based on a Stokes-type expansion for surface elevation and a scaling argument for velocity potential. Third-order correction of the initial conditions may improve the accuracy of the solution and eliminate third-order error waves which manifest as superharmonics and principal harmonics, described by the Stokes expansion in Eq. (7). The efficacy of the third-order correction has been analysed with the third-order superharmonic error waves. If no thirdorder correction is performed, the third-order bound harmonics will be generated during the course of the simulation accompanied by the generation of third-order free harmonics, known as error waves, required to satisfy the governing equations. The third-order superharmonic error waves possess a wavenumber substantially higher than the free harmonics comprising the wave group. Thus, the third-order superharmonic error waves will eventually lag behind and separate out from the main group as a result of the lower phase velocity. Figure 8 depicts the surface elevation along the center plane of the wave group after nonlinear focus, $t=12 T_{p}$, for a solution with exact second-order correction of the initial conditions and another solution with exact second-order correction and approximate third-order correction of the initial conditions using the proposed scheme. As can be seen, the third-order superharmonic error waves have separated out from the main group for the case of second-order initial conditions. In contrast, the solution with approximately thirdorder initial conditions exhibits no discernible third-order superharmonic error waves confirming the efficacy of the proposed correction. Note, however, that a similar assessment of the thirdorder principal harmonic error waves cannot be performed since these error waves propagate with the wave group and do not separate out during the course of the simulation. 


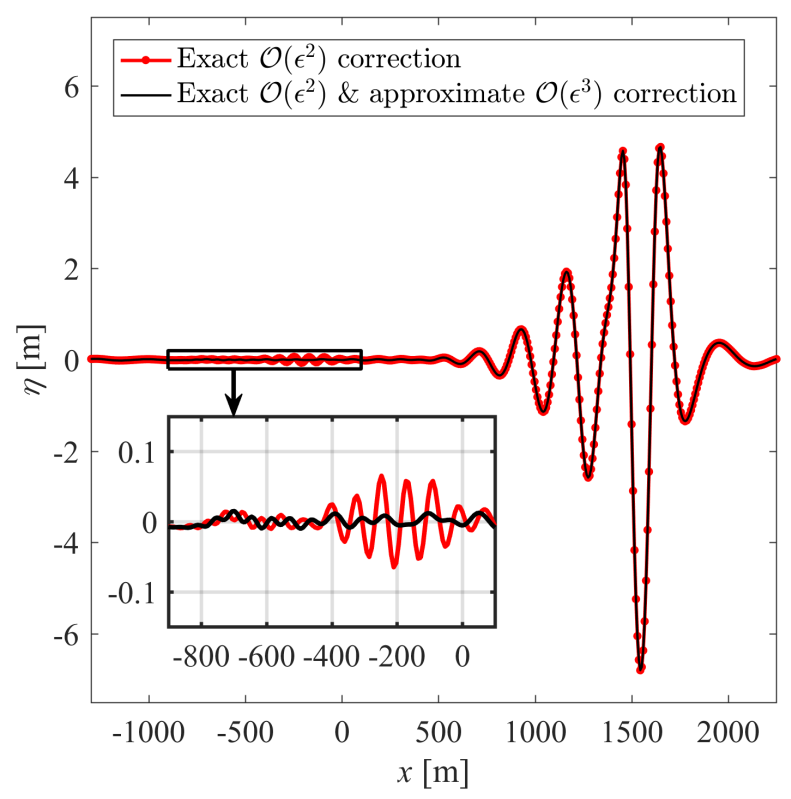

FIGURE 8. APPROXIMATE THIRD-ORDER CORRECTION: surface elevation along center plane of wave group at time $t=12 T_{p}$ confirms that third-order superharmonic error waves have been eliminated.

\section{DISCUSSION}

The spectral evolution depicted in Fig. 5 has been attributed to resonant third-order interactions, based on the simulations of Gibbs \& Taylor [8] which utilised an expanded form of DirichletNeumann operator (the $G$-operator) to capture various orders of nonlinear wave-wave interactions. The third-order version of the $G$-operator produced results similar to Fig. 5 confirming resonant third-order wave-wave interactions as the cause of the spectral evolution. A notable feature of the spectral evolution in Fig. 5 is the development of low-wavenumber and high-wavenumber sidelobes in the initially narrow-banded wavenumber spectrum, with an obvious bias in energy transfer to high-wavenumber components. The high-wavenumber sidelobe initially develops at an angle of $\pm 35 \mathrm{deg}$ to the spectral peak up until the time of nonlinear focus, corresponding to the $\arctan (1 / \sqrt{ } 2)$ resonance angle identified by Longuet-Higgins [12] for the spectral peak of a narrow-banded three-dimensional wave packet and the resonance angle predicted by the Phillips "figure-of-eight" loop [23] for the narrow-banded interactions of a degenerate quartet. Agreement with the narrow-banded results of Longuet-Higgins [12] and Phillips [23] may be expected in the pre-focus regime since the wavenumber spectrum remains narrow-banded up until nonlinear focus (Fig. 5(b)). However, after focus, the wavenumber spectrum broadens and the high-wavenumber sidelobe shifts, ultimately forming an angle of $\pm 55 \mathrm{deg}$ with the spectral peak. Energy transfer to high-wavenumber components propagating at an oblique angle to the spectral peak has, thus, been confirmed by the present study and may be responsible for the formation of obliquely propagating wing waves observed during the simulated extreme wave event. Contour plots of surface elevation are shown in Fig. 9 in the post focus regime $\left(t>1.3 T_{p}\right)$; only crests are shown since the contour levels are evenly distributed between $1 \mathrm{~m}$ and $11 \mathrm{~m}$ in intervals of $1 \mathrm{~m}$. The surface elevation is shown on both sides of the symmetry plane for clarity and the results are plotted in a reference frame that moves with the group velocity in the direction of group propagation. Crest $\mathcal{C} 0$ leads the group at $t=5.1 T_{p}$, shown in Figure 9(a), and exhibits the highest surface elevation of all the crests - the position of the largest crest at the front of the wave group is consistent with the observations of Adcock \& Taylor [11] as well as Gibbs \& Taylor [8]. Figure $9(a)$ also depicts crest $\mathcal{C} 1$ trailing behind the leading crest and a wing wave $\mathcal{W} 1$ appears at the periphery of the wave group. A gradual merger of the trailing crest $\mathcal{C} 1$ with the wing wave $\mathcal{W} 1$ is depicted at $t=5.5 T_{p}$ in Fig. $9(b)$ and at $t=5.9 T_{p}$ in Fig. $9(c)$. The merger process occurs as crest $\mathcal{C} 1$ moves towards the front of the wave group, gradually overtaking the wing wave $\mathcal{W} 1$, while the the leading crest $\mathcal{C} 0$ diminishes in amplitude. The wing wave $\mathcal{W} 1$ can, thus, be seen to propagate slower than the crest $\mathcal{C} 1$ in the mean direction of wave group propagation-an expected result since the formation of wing waves is associated with energy transfer to high-wavenumber components propagating at an oblique angle to the spectral peak. Crest $\mathcal{C} 1$ has overtaken the wing wave $\mathcal{W} 1$ at $t=6.3 T_{p}$, shown in Fig. $9(d)$, and another wing wave $\mathcal{W} 2$ appears at the periphery of the wave group at $t=6.7 T_{p}$, shown in Fig. 9(e). The distance between the wing waves $\mathcal{W} 1$ and $\mathcal{W} 2$ is smaller than the distance between the central crests, consistent with the high-wavenumber components thought to comprise the wing waves. Complete merger of the wing wave $\mathcal{W} 1$ with the central crest $\mathcal{C} 1$ can be seen at $t=7.1 T_{p}$ in Fig. $9(f)$ forming a single, crescent-shaped, broad crest at the front of the wave group while crest $\mathcal{C} 0$, which initially led the group, has completely receded and no longer appears in the plot. The present study has, thus, numerically simulated a narrow-banded extreme wave event and observed energy transfer to high-wavenumber components propagating at an oblique angle to the spectral peak. The preferential energy transfers have been attributed to third-order resonant interactions and associated with the formation of wing waves at the periphery of the wave group. Constructive interference between the wing waves and central crests contributes to the formation of broad, crescentshaped crests at the front of the wave group_-previously termed "walls of water" [8]. Thus, directional energy transfer due to third-order interactions has influenced the shape of the narrowbanded extreme wave event simulated in this study.

\section{CONCLUSION}

The current study has investigated the spectral evolution of a steep, focusing wave group using the fully-nonlinear potential flow solver OceanWave3D with a detailed assessment of simulation fidelity. A combination of eighth-order finite differencing 
(a)

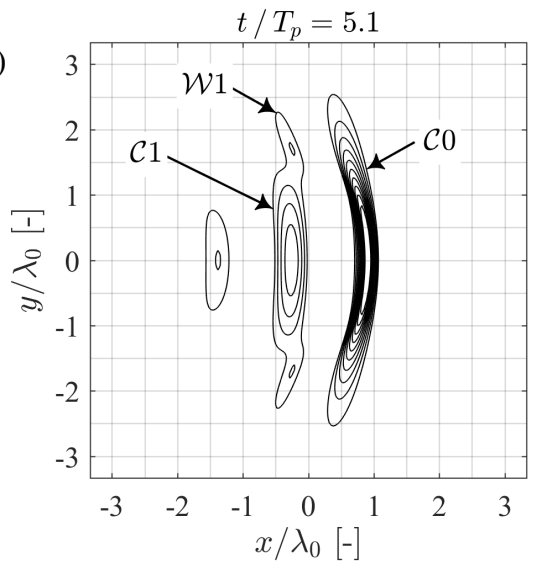

(d)

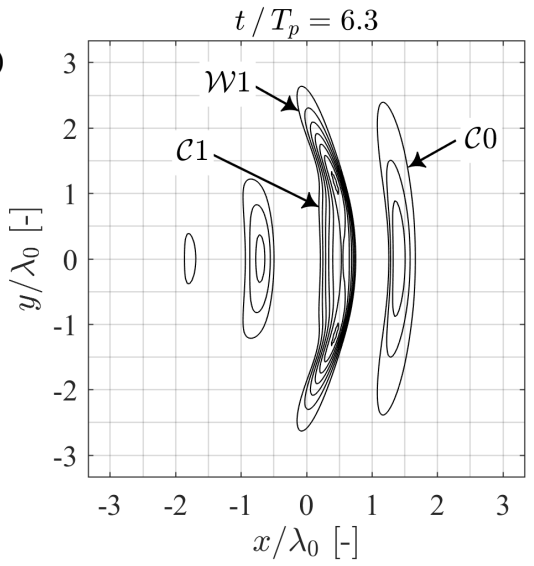

(b)

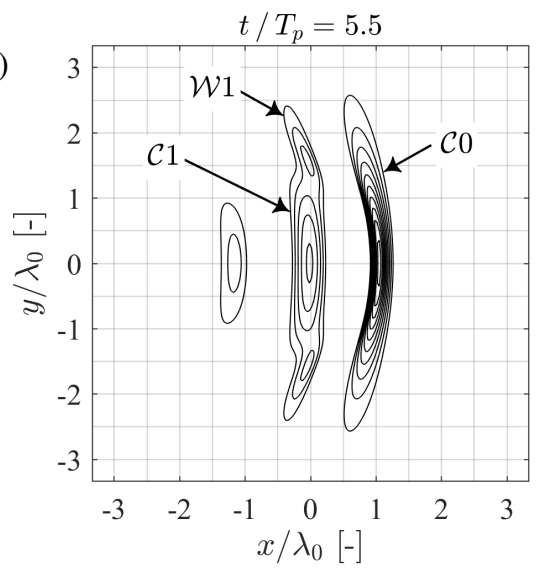

(e)

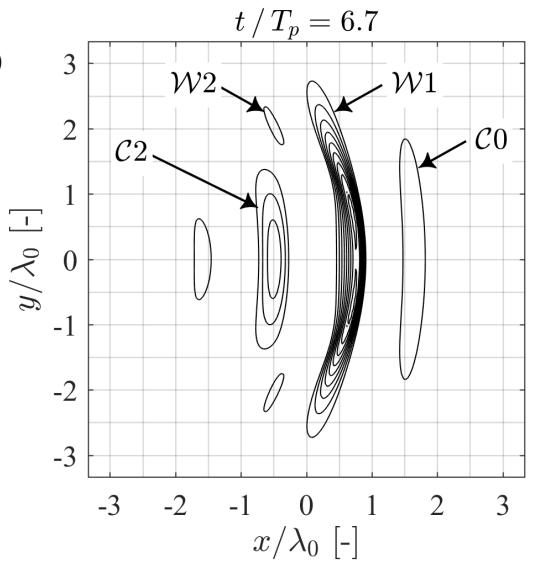

(c)

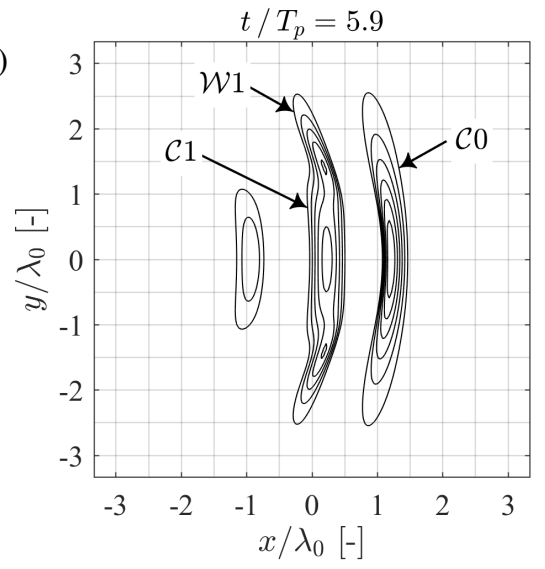

$(f)$

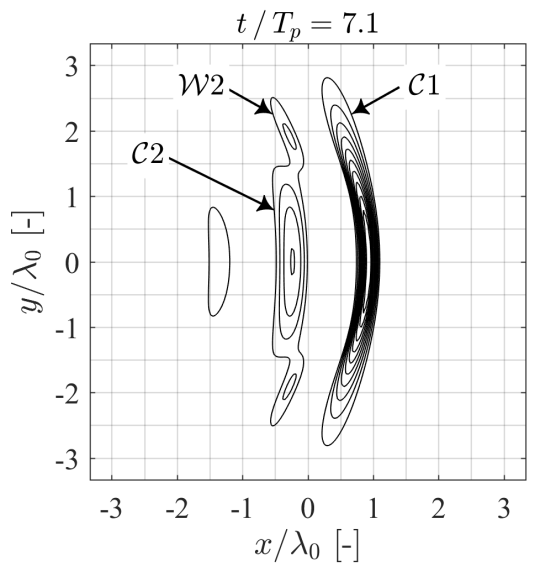

FIGURE 9. Contour plots of surface elevation evenly distributed between $1 \mathrm{~m}$ and $11 \mathrm{~m}$ in intervals of $1 \mathrm{~m} —$ only crests are shown: $(a) t / T_{p}=5.1$; (b) $t / T_{p}=5.5 ;(c) t / T_{p}=5.9 ;(d) t / T_{p}=6.3 ;(e) t / T_{p}=6.7 ;(f) t / T_{p}=7.1$.

with a spatial resolution of 30 grid points per characteristic wavelength, in the the direction of group propagation, and a CourantFriedrichs-Lewy (CFL) condition of 0.5 has been used to validate the simulations against a Higher-Order Spectral (HOS) code and achieve energy conservation within $0.024 \%$. A symmetry plane located along the center plane of the wave group has proved effective in reducing the size of the numerical domain, with negligible aggravation of the numerical diffusion, and the finite-depth domain with $k_{p} d=5.6$ has been found to be a reasonable approximation for infinitely deep water. A novel approximate thirdorder correction scheme for the initial conditions has been proposed and shown to eliminate the third-order superharmonic error waves. Negligible aliasing error has also been confirmed by running simulations forwards in time, with a positive time step, and backwards in time, with a negative time step, to recover the initial condition and ensure that all spectral evolution is the result of physical processes. Energy transfer to high-wavenumber components propagating at an angle of approximately $\pm 35 \mathrm{deg}$ to the spectral peak has been confirmed, up until the time of nonlinear focus, which may be responsible for the obliquely propagating "wing waves" observed in the simulated extreme wave event.

\section{ACKNOWLEDGMENT}

The authors gratefully acknowledge funding from the DeRisk project of Innovation Fund Denmark (grant number 4106-00038B) and also express gratitude to the DTU Computing Center (DCC) for use of the High Performance Computing (HPC) clusters. DB has been supported by a studentship from the Engineering and Physical Sciences Research Council (EPSRC) of the UK Government and would like to specifically thank Professor Paul H. Taylor for useful discussions regarding the numerical simulations.

\section{REFERENCES}

[1] Kharif, C., and Pelinovsky, E., 2003, "Physical mechanisms of the rogue wave phenomenon," Eur. J. Mech. B/Fluids, 22, pp. 603-634.

[2] Dysthe, K., Krogstad, H. E., and Müller, P., 2008, "Oceanic rogue waves," Annu. Rev. Fluid Mech., 40, pp. 287-310.

[3] Adcock, T. A. A., and Taylor, P. H., 2014, "The physics of anomalous ('rogue') ocean waves," Rep. Prog. Phys., 77: 105901. 
[4] Adcock, T. A. A., Taylor, P. H., and Draper, S., 2015, "Nonlinear dynamics of wave-groups in random seas: unexpected walls of water in the open ocean," Proc. R. Soc. A, 471: 20150660.

[5] Fujimoto, W., Waseda, T., and Webb, A., 2018, "Impact of the four-wave quasi-resonance on freak wave shapes in the ocean," Ocean Dynamics, DOI: 10.1007/s10236-0181234-9.

[6] Latheef, M., Swan, C., and Spinneken, J., 2017, "A laboratory study of nonlinear changes in the directionality of extreme seas," Proc. R. Soc. A, 473: 20160290.

[7] Bateman, W. J. D., Katsardi, V., and Swan, C., 2012, "Extreme ocean waves. Part I. The practical application of fully nonlinear wave modelling," Appl. Ocean Res., 34, pp. 209224.

[8] Gibbs, R. H., and Taylor, P. H., 2005, "Formation of walls of water in 'fully' nonlinear simulations," Appl. Ocean Res., 27, pp. 142-157.

[9] Tromans, P. S., Anaturk, A., and Hagemeijer, P., 1991, "A new model for the kinematics of large ocean wavesapplication as a design wave," Proceedings of the first international offshore and polar engineering conference (ISOPE), Edinburgh UK, Aug. 11-16.

[10] Bateman, W. J. D., Swan, C., and Taylor, P. H., 2001, “On the efficient numerical simulation of directionally spread surface water waves," J. Comp. Phys., 174, pp. 277-305.

[11] Adcock, T. A. A., and Taylor, P. H., 2016, "Fast and local non-linear evolution of steep wave-groups on deep water: A comparison of approximate models to fully nonlinear simulations," Phys. Fluids, 28: 016601.

[12] Longuet-Higgins, M. S., 1976, "On the nonlinear transfer of energy in the peak of a gravity-wave spectrum: a simplified model," Proc. R. Soc. Lond. A., 347, pp. 311-328.

[13] Engsig-Karup, A. P., Bingham, H. B., and Lindberg, O., 2009, "An efficient flexible-order model for 3D nonlinear water waves," J. Comp. Phys., 228, pp. 2100-2118.

[14] Currie, I. G., 1993, Fundamental Mechanics of Fluids (second edition), McGraw-Hill, pp. 201-204.

[15] Bingham, H. B., and Zhang, H., 2007, "On the accuracy of finite-difference solutions for nonlinear water waves," J. Eng. Math, 58, pp. 211-228.

[16] Dalzell, J. F., 1999, "A note on finite depth second-order wave-wave interactions," Appl. Ocean Res., 21, pp. 105111.

[17] Walker, D. A. G., Taylor, P. H., and Eatock Taylor, R., 2004, "The shape of large surface waves on the open sea and the Draupner New Year Wave," Appl. Ocean Res., 26, pp. 7383.

[18] Fitzgerald, C. J., Taylor, P. H., Eatock Taylor, R., Grice, J., and Zhang, J., 2014, "Phase manipulation and the harmonic components of ringing forces on a surface-piercing column,” Proc. R. Soc. A, 470: 20130847.
[19] Madsen, P. A. and Fuhrman, D. R., 2012, "Third-order theory for multi-directional irregular waves," J. Fluid Mech., 698, pp. 304-334.

[20] Lannes, D., 2013, "The water waves problem: mathematical analysis and asymptotics," American Mathematical Society, Mathematical Surveys and Monographs, 188, p. 16.

[21] Ducrozet, G., Bingham, H. B., Engsig-Karup, A. P., Bonnefoy, and F., Ferrant, P., 2012, "A comparative study of two fast nonlinear free-surface water wave models," Int. J. Numer. Meth. Fluids, 69, pp. 1818-1834.

[22] van den Bremer, T. S., and Taylor, P. H., 2015, "Estimates of Lagrangian transport by surface gravity wave groups: The effects of finite depth and directionality," J. Geophys. Res. Oceans, 120, pp. 2701-2722.

[23] Phillips, O. M., 1960, "On the dynamics of unsteady gravity waves of finite amplitude. Part 1 . The elementary interactions,” J. Fluid Mech., 9(2), pp. 193-217.

\section{Appendix A: Pseudo-Second-Order Error Waves}

The exact second-order theory of Dalzell [16] provides a finite-depth expression for the first-order velocity potential, $\phi^{(1)}$, resulting from two interacting wave trains $(j=1$ and $j=2)$ :

$$
\phi^{(1)}=\sum_{j=1}^{2} a_{j} \frac{g}{\omega_{j}} \frac{\cosh \left\{\left|k_{j}\right|(z+d)\right\}}{\cosh \left\{\left|k_{j}\right| d\right\}} \sin \psi_{j},
$$

where $a_{j}, \omega_{j}, k_{j}$ and $\psi_{j}$ denote the component amplitude, angular frequency, wavenumber and phase respectively.

Reformulation of the hyperbolic functions followed by a first order Taylor series expansion about $z=0$ and invocation of the linear dispersion relation, $\omega_{j}^{2}=g\left|k_{j}\right| \tanh \left\{\left|k_{j}\right| d\right\}$, yields:

$$
\begin{aligned}
\left.\phi^{(1)}\right|_{z=\eta} & =\left.\phi^{(1)}\right|_{z=0} \\
& +\sum_{j=1}^{2} \sum_{k=1}^{2} \frac{1}{2} a_{j} a_{k} \omega_{j} \sin \left\{\psi_{j}+\psi_{k}\right\} \\
& +\sum_{j=1}^{2} \sum_{k=1}^{2} \frac{1}{2} a_{j} a_{k} \omega_{j} \sin \left\{\psi_{j}-\psi_{k}\right\}
\end{aligned}
$$

Calculation of the first order initial conditions at the mean-waterlevel $(z=0)$ rather than the free surface $(z=\eta)$, thus, results in spurious terms which resemble second-order superharmonics and subharmonics in form-manifesting as pseudo-second-order error waves in the OceanWave $3 D$ simulations. 